\title{
Gaussian fluctuations of polarization in the region of phase transitions in DMAGaS-DMAAIS ferroelectrics in the framework of the four-state model
}

\author{
I.V.Stasyuk, O.V.Velychko \\ Institute for Condensed Matter Physics \\ of the National Academy of Sciences of Ukraine, \\ 1 Svientsitskii Str., 79011 Lviv, Ukraine
}

Received 12 November, 2003

\begin{abstract}
A description of thermodynamics of the DMAGaS-DMAAIS family ferroelectrics improved by taking into account the Gaussian fluctuations of polarization is presented. Fluctuations become important in the vicinity of the second order (or the first order close to the second order) phase transitions which is the case of the mentioned crystals. The more elaborated theory, adapting the Onyszkiewicz approach to the purpose of the four-state model, provides equations for components of polarization, their fluctuations and free energy in a simple form with a clear physical sense. The results obtained by numerical calculations demonstrate that in the considered system at the sufficiently long-ranged interaction the Gaussian fluctuations lead to the slight decrease of temperatures of phase transitions from paraphase to ferrophase and in the region of the triple point they are of the same order of magnitude for both first and second order phase transitions.
\end{abstract}

Key words: ferroelectrics, DMAGaS, DMAAIS, microscopic model, Gaussian fluctuations

PACS: 77.84.-s, 64.60.Cn

\section{Introduction}

The present work is devoted to the investigation of thermodynamics of complex order-disorder systems on the basis of the four-state model. Such a model was proposed earlier [1] for a description of dielectric properties of the ferroelectric crystals $\left(\mathrm{CH}_{3}\right)_{2} \mathrm{NH}_{2} \mathrm{Me}\left(\mathrm{SO}_{4}\right)_{2} \cdot 6 \mathrm{H}_{2} \mathrm{O}(\mathrm{Me}=\mathrm{Al}, \mathrm{Ga})$ (dimethylammonium aluminium (or gallium) sulphate hexahydrate - abbreviated as DMAAlS or DMAGaS, respectively) [2-4]. The model can also be used in order to consider of the phase transitions in crystals $\mathrm{KHCO}_{3}$ and $\mathrm{KDCO}_{3}$, the proton rearrangements in the trimers $\mathrm{H}_{2}\left(\mathrm{SeO}_{4}\right)$ in the crystals $\left(\mathrm{NH}_{4}\right)_{4} \mathrm{H}_{2}\left(\mathrm{SeO}_{4}\right)$ as well as the correlated anharmonic motion in the 
pairs of the neighbouring apex oxygen ions in the high- $T_{\mathrm{c}}$ superconducting systems of the $\mathrm{YBa}_{2} \mathrm{Cu}_{3} \mathrm{O}_{7-\delta}$ type. In the framework of the four-state model the sequential phase transitions from paraelectric to ferroelectric and antiferroelectric states in the DMAGaS and DMAAIS crystals during the lowering of temperature were described taking into account the spontaneous ordering of dimethylammonium (DMA) groups [5]. Each DMA group can be oriented in four different ways; the orientational states differ in pairs in their energy. The results obtained in the mean field approximation (MFA), such as the temperature behaviour of spontaneous polarization, the changes in occupation of the orientational states and phase diagrams [5], are in satisfactory agreement with the experimental data $[2-4,6]$. The effect of suppression of the ferroelectric phase in DMAGaS under the influence of hydrostatic pressure [7] was also explained by the model.

The obtained phase diagrams show that the paraelectric-ferroelectric phase transition in the DMAGaS crystal is of the first order close to the second order (and close to the tricritical point) [5]. It is also situated near the triple point where the para-, ferro-, and antiferroelectric phases coexist. In the case of the DMAAlS crystal the situation is slightly different. The presence of only one phase transition (between para- and ferroelectric phases) is indubitably established. It corresponds to another cross-section on the phase diagram for the systems of this type.

The shape of the phase diagrams and the absolute values of the phase transition temperatures can change when we go beyond the MFA. Usually the fluctuation effects, growing up near the transition point, lead to the decrease of $T_{\mathrm{c}}$. Their role in the case of the four-state model is not yet elucidated, especially in the vicinity of the tricritical point.

The fluctuations of the order parameter (which determines the spontaneous polarization of the crystal and in the considered case is connected with the differences in occupations of the orientational states) can be taken into account in the simplest way within the Gaussian approximation. According to this approach, in the present work we will perform an investigation of the effect of fluctuation using the scheme proposed for the Ising model and developed for models of this class by Onyszkiewicz [8]. The scheme is based on the thermodynamically consistent description of quadratic fluctuations (with respect to the MFA mean values) by self-consistent determination of the variation of the corresponding Gaussian distribution. We will generalize this approach on the case of the four-state model. The calculation of the temperature dependences of these characteristics and estimation of their magnitudes will be performed. As a specific example, the application of the model to the description of phase transitions in the DMAGaS and DMAAlS crystals is considered. 


\section{Hamiltonian of the four-state model: mean field and fluctua- tion parts}

According to the four-state model [5], the Hamiltonian of the subsystem of DMA groups constructed on the basis of their four orientational states looks as follows:

$$
H=\sum_{n k} \sum_{s} \lambda_{k s} X_{n k}^{s s}-\frac{1}{2} \sum_{n n^{\prime}} \sum_{k k^{\prime}} \sum_{\alpha \alpha^{\prime}} \Psi_{k k^{\prime}}^{\alpha \alpha^{\prime}}\left(n n^{\prime}\right) D_{n k}^{\alpha} D_{n^{\prime} k^{\prime}}^{\alpha^{\prime}}
$$

where

$$
\begin{aligned}
& \lambda_{k 1}=\lambda_{k 2}=\varepsilon_{1}, \quad \lambda_{k 3}=\lambda_{k 4}=\varepsilon_{2}, \\
& D_{n k}^{x}=d_{x}\left(X_{n k}^{22}-X_{n k}^{11}\right), \quad D_{n k}^{y}=d_{y}\left(X_{n k}^{44}-X_{n k}^{33}\right), \quad D_{n k}^{z}=0 .
\end{aligned}
$$

The Hubbard operator $X_{n k}^{s s}=|n k, s\rangle\langle n k, s|$ describes the DMA complex residing in the orientational state $s(s=1, \ldots, 4)$ at the lattice site $n$ and the sublattice $k(k=1,2), d_{\alpha}$ is the magnitude (defined by the structure of the crystal) of the $\alpha$-component $D_{n k}^{\alpha}$ of the dipole moment of the complex, $\Psi_{k k^{\prime}}^{\alpha \alpha^{\prime}}\left(n n^{\prime}\right)$ is the energy of the dipole interaction, $\varepsilon_{1}$ and $\varepsilon_{2}$ are the energies of the DMA groups in the positions $(k, 1),(k, 2)$ and $(k, 3),(k, 4)$, respectively. Here the possibility of reorientational hopping of DMA groups is neglected.

Hamiltonian (1) can be separated into two parts:

$$
H=H_{\mathrm{MFA}}+H^{\prime},
$$

where the mean field term is equal to

$$
\begin{aligned}
H_{\mathrm{MFA}} & =N U_{0}+\sum_{n k} \sum_{s} \lambda_{k s} X_{n k}^{s s}-\sum_{n k} \sum_{\alpha} F_{k}^{\alpha} D_{n k}^{\alpha}, \\
U_{0} & =\frac{1}{2} \sum_{k k^{\prime}} \sum_{\alpha \alpha^{\prime}} \psi_{k k^{\prime}}^{\alpha \alpha^{\prime}}\left\langle D_{k}^{\alpha}\right\rangle\left\langle D_{k^{\prime}}^{\alpha^{\prime}}\right\rangle, \\
F_{k}^{\alpha} & =\sum_{k^{\prime}} \sum_{\alpha^{\prime}} \psi_{k k^{\prime}}^{\alpha \alpha^{\prime}}\left\langle D_{k^{\prime}}^{\alpha^{\prime}}\right\rangle, \\
\psi_{k k^{\prime}}^{\alpha \alpha^{\prime}} & =\sum_{n^{\prime}} \Psi_{k k^{\prime}}^{\alpha \alpha^{\prime}}\left(n n^{\prime}\right)
\end{aligned}
$$

and the explicit structure of the matrix $\psi_{k k^{\prime}}^{\alpha \alpha^{\prime}}$, which is the Fourier transform of the interaction matrix at $\mathbf{q}=0$, looks like

$$
\hat{\psi}=\left(\begin{array}{cccccc}
a & b & c & d & e & f \\
b & g & h & -e & k & l \\
c & h & m & f & -l & n \\
d & -e & f & a & -b & c \\
e & k & -l & -b & g & -h \\
f & l & n & c & -h & m
\end{array}\right)
$$


the matrix elements $a, \ldots, n$ are considered to be the parameters of the dipoledipole interaction. Here the rows and columns are numbered by a composite index $\{k \alpha\}=\{1 x, 1 y, 1 z, 2 x, 2 y, 2 z\}$. The fluctuation part is given by

$$
H^{\prime}=-\frac{1}{2} \sum_{n n^{\prime}} \sum_{k k^{\prime}} \sum_{\alpha \alpha^{\prime}} \Psi_{k k^{\prime}}^{\alpha \alpha^{\prime}}\left(n n^{\prime}\right)\left(D_{n k}^{\alpha}-\left\langle D_{k}^{\alpha}\right\rangle\right)\left(D_{n^{\prime} k^{\prime}}^{\alpha^{\prime}}-\left\langle D_{k^{\prime}}^{\alpha^{\prime}}\right\rangle\right) .
$$

(we do not consider here the phase transitions which are accompanied by the change of the lattice period). The Hamiltonians $H_{\mathrm{MFA}}$ and $H^{\prime}$ commute; so the average projections of dipole moments can be expressed with the allowance for fluctuations as

$$
\left\langle D_{k}^{\alpha}\right\rangle=\left\langle D_{k}^{\alpha} \mathrm{e}^{-\beta H^{\prime}}\right\rangle_{0} /\left\langle\mathrm{e}^{-\beta H^{\prime}}\right\rangle_{0}
$$

where the averaging $\langle\ldots\rangle_{0}$ is done in the MFA

$$
\langle\ldots\rangle_{0}=\operatorname{Tr}\left(\ldots \mathrm{e}^{-\beta H_{\mathrm{MFA}}}\right) / \operatorname{Tr} \mathrm{e}^{-\beta H_{\mathrm{MFA}}}, \quad \beta=1 / \Theta=1 / k_{\mathrm{B}} T .
$$

The expansion of the right-hand side of expression (7) into the power series with respect to $H^{\prime}$ and the averaging of each term are performed in the framework of technique [9] with the help of semi-invariants built on $X$-operators. The ovals encircling the sites of diagonal $X$-operators correspond to semi-invariants in the diagrammatic representation; the components $\psi_{k k^{\prime}}^{\alpha \alpha^{\prime}}(\mathbf{q})$ are represented by the interaction lines.

In our case all diagrams, containing the parts without external vertices connected to the rest of the diagram by a single line (single-tailed diagrams), are already included in the zero approximation. So, every connected diagram in our expansion is formed by the blocks (ovals) containing external vertices with additional elements connected to them by two, three etc. interaction lines (polytailed diagrams). We restrict our study to the double-tailed diagrams which allows one to take into account the fluctuations of the mean field in the form of the Gaussian distribution (for the case of the simple Ising model, such an approach is analyzed more in detail in [10]).

The simplest approximation (when only the first term of the series expansion for the pair $\langle X X\rangle$-correlator from the double-tailed diagram is taken into account) leads to nonphysical results. For this reason we base our study on the Onyszkiewicz approach [8] where the $\langle X X\rangle$-correlators in the double-tailed diagrams are selfconsistently calculated at each stage of derivation of equations for the mean values of dipole moments $\left\langle D_{k}^{\alpha}\right\rangle$ and Gaussian fluctuation parameters as well as on the evaluation of free energy. Owing to this procedure the theory becomes internally consistent.

\section{Equations for components of dipole moments in the double- tailed diagram approach}

Lets us write the series for the mean value of the dipole moment component $\left\langle D_{k}^{\alpha}\right\rangle$ including contributions of two-tailed parts of diagrams. In the diagrammatic notation [8] it looks like

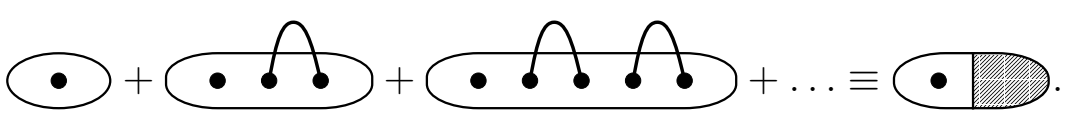


A corresponding analytic expression is

$$
\begin{aligned}
\left\langle D_{k}^{\alpha}\right\rangle= & b_{k}^{\alpha}\left(y_{k}^{x}, y_{k}^{y}\right)+\frac{1}{1 !} \sum_{\alpha_{1} \alpha_{2}} j_{k k}^{\alpha_{1} \alpha_{2}} \frac{\partial^{2}}{\partial\left(\beta y_{k}^{\alpha_{1}}\right) \partial\left(\beta y_{k}^{\alpha_{2}}\right)} b_{k}^{\alpha}\left(y_{k}^{x}, y_{k}^{y}\right) \\
& +\frac{1}{2 !} \sum_{\alpha_{1} \alpha_{2}} \sum_{\alpha_{3} \alpha_{4}} j_{k k}^{\alpha_{1} \alpha_{2}} j_{k k}^{\alpha_{3} \alpha_{4}} \frac{\partial^{4}}{\partial\left(\beta y_{k}^{\alpha_{1}}\right) \ldots \partial\left(\beta y_{k}^{\alpha_{4}}\right)} b_{k}^{\alpha}\left(y_{k}^{x}, y_{k}^{y}\right)+\ldots
\end{aligned}
$$

where

$$
b_{k}^{\alpha}\left(y_{k}^{x}, y_{k}^{y}\right)=\frac{\partial L_{k}}{\partial\left(\beta y_{k}^{\alpha}\right)}
$$

is the generating function, which also determines the contribution to the $\left\langle D_{k}^{\alpha}\right\rangle$ mean value in the MFA. Here $L_{k}=\ln Z_{k}$, and

$$
Z_{k}=2 \mathrm{e}^{-\beta \varepsilon}\left[\mathrm{e}^{\beta \Delta} \cosh \left(\beta d_{x} y_{k}^{x}\right)+\mathrm{e}^{-\beta \Delta} \cosh \left(\beta d_{y} y_{k}^{y}\right)\right]
$$

is the partition function in MFA $\left(\varepsilon=\left(\varepsilon_{1}+\varepsilon_{2}\right) / 2, \Delta=\left(\varepsilon_{2}-\varepsilon_{1}\right) / 2\right)$. The internal fields $y_{k}^{\alpha}=\sum_{k^{\prime} \alpha^{\prime}} \psi_{k k^{\prime}}^{\alpha \alpha^{\prime}}\left\langle D_{k^{\prime}}^{\alpha^{\prime}}\right\rangle$ are introduced, namely

$$
\begin{aligned}
& y_{1}^{x}=a\left\langle D_{1}^{x}\right\rangle+d\left\langle D_{2}^{x}\right\rangle+b\left\langle D_{1}^{y}\right\rangle+e\left\langle D_{2}^{y}\right\rangle, \\
& y_{1}^{y}=b\left\langle D_{1}^{x}\right\rangle-e\left\langle D_{2}^{x}\right\rangle+g\left\langle D_{1}^{y}\right\rangle+k\left\langle D_{2}^{y}\right\rangle, \\
& y_{2}^{x}=d\left\langle D_{1}^{x}\right\rangle+a\left\langle D_{2}^{x}\right\rangle-e\left\langle D_{1}^{y}\right\rangle-b\left\langle D_{2}^{y}\right\rangle, \\
& y_{2}^{y}=e\left\langle D_{1}^{x}\right\rangle-b\left\langle D_{2}^{x}\right\rangle+k\left\langle D_{1}^{y}\right\rangle+g\left\langle D_{2}^{y}\right\rangle .
\end{aligned}
$$

At last, the matrix

$$
j_{k k}^{\alpha_{1} \alpha_{2}}=\frac{1}{N} \sum_{\mathbf{q}} \widetilde{I}_{k k}^{\alpha_{1} \alpha_{2}}(\mathbf{q}),
$$

denoted by the solid line in the diagram representation, describes the contribution of the two-tailed parts (its explicit form will be discussed below). After applying the Fourier transformation the infinite series in expression (10) can be summed up [10]

$$
\begin{aligned}
\left\langle D_{k}^{\alpha}\right\rangle= & \int_{-\infty}^{+\infty}\left[\frac{1}{(2 \pi)^{2}} \int_{-\infty}^{+\infty} \mathrm{e}^{\mathrm{i}\left(y_{k}^{x}-z_{1}\right) \tau_{1}} \mathrm{e}^{\mathrm{i}\left(y_{k}^{y}-z_{2}\right) \tau_{2}} \exp \left(-\sum_{\alpha_{1} \alpha_{2}} j_{k k}^{\alpha_{1} \alpha_{2}} \tau_{\alpha_{1}} \tau_{\alpha_{2}}\right) \mathrm{d} \tau_{1} \mathrm{~d} \tau_{2}\right] \\
& \times b_{k}^{\alpha}\left(z_{1}, z_{2}\right) \mathrm{d} z_{1} \mathrm{~d} z_{2} .
\end{aligned}
$$

Let us substitute $\tau_{\alpha}$ by new variables $\bar{\tau}_{\mu}$

$$
\tau_{\alpha}=\sum_{\mu} u_{\alpha \mu}^{k} \bar{\tau}_{\mu}
$$

where $\hat{U}_{k}=u_{\alpha \mu}^{k}$ is the matrix of unitary transformation reducing the matrix $\hat{J}_{k}=$ $\left[j_{k k}^{\alpha_{1} \alpha_{2}}\right]$ to the diagonal form with eigenvalues $\lambda_{1}$ and $\lambda_{2}$. Now the double integral over the variables $\bar{\tau}_{1}$ and $\bar{\tau}_{2}$ splits onto independent parts and can be written as

$$
\prod_{\mu=1}^{2}\left[\frac{1}{2 \pi} \int_{-\infty}^{+\infty} \mathrm{e}^{\mathrm{i} w_{\mu}^{k} \bar{\tau}_{\mu}} \mathrm{e}^{-\lambda_{\mu}^{k} \bar{\tau}_{\mu}^{2}} \mathrm{~d} \bar{\tau}_{\mu}\right]=\prod_{\mu=1}^{2}\left[\frac{1}{2 \sqrt{\pi \lambda_{\mu}^{k}}} \exp \left(-\frac{\left(w_{\mu}^{k}\right)^{2}}{4 \lambda_{\mu}^{k}}\right)\right],
$$


where

$$
w_{\mu}^{k}=\sum_{\alpha}\left(y_{k}^{\alpha}-z_{\alpha}\right) u_{\alpha \mu}^{k} .
$$

Respectively, expression (14) looks like

$$
\left\langle D_{k}^{\alpha}\right\rangle=\prod_{\mu=1}^{2} \frac{1}{2 \sqrt{\pi \lambda_{\mu}^{k}}} \int_{-\infty}^{+\infty} \exp \left(-\frac{\left(w_{\mu}^{k}\left(z_{1}, z_{2}\right)\right)^{2}}{4 \lambda_{\mu}^{k}}\right) b_{k}^{\alpha}\left(z_{1}, z_{2}\right) \mathrm{d} z_{1} \mathrm{~d} z_{2},
$$

or, making use of the identity $z_{\alpha}=y_{k}^{\alpha}-\sum_{\mu} w_{\mu}^{k} u_{\alpha \mu}^{k}$, we can transform it to the form

$$
\begin{aligned}
\left\langle D_{k}^{\alpha}\right\rangle= & \prod_{\mu=1}^{2} \frac{1}{2 \sqrt{\pi \lambda_{\mu}^{k}}} \int_{-\infty}^{+\infty} \exp \left(-\frac{\left(w_{\mu}^{k}\right)^{2}}{4 \lambda_{\mu}^{k}}\right) \\
& \times b_{k}^{\alpha}\left(y_{k}^{x}-\sum_{\eta_{1}} u_{1 \eta_{1}}^{k} w_{\eta_{1}}^{k}, y_{k}^{y}-\sum_{\eta_{2}} u_{2 \eta_{2}}^{k} w_{\eta_{2}}^{k}\right) \mathrm{d} w_{1}^{k} \mathrm{~d} w_{2}^{k} .
\end{aligned}
$$

The last expression could be made more physically transparent when the fluctuations of the mean field component will be presented in an explicit form. Let us use the relations

$$
\prod_{\mu} \lambda_{\mu}^{k}=\operatorname{det}\left[j_{k k}^{\alpha \alpha^{\prime}}\right]=\mathcal{D}_{k}, \quad \sum_{\mu} \frac{\left(w_{\mu}^{k}\right)^{2}}{4 \lambda_{\mu}^{k}}=\frac{1}{4} \sum_{\alpha \alpha^{\prime}} A_{k k}^{\alpha \alpha^{\prime}}\left(y_{k}^{\alpha}-z_{\alpha}\right)\left(y_{k}^{\alpha^{\prime}}-z_{\alpha^{\prime}}\right)
$$

where the matrix $\hat{A}_{k}$, inverse to the $\hat{J}_{k}$ matrix, is introduced

$$
A_{k k}^{\alpha \alpha^{\prime}} \equiv\left(\hat{J}_{k}^{-1}\right)_{\alpha \alpha^{\prime}}=\sum_{\mu} u_{\alpha \mu}^{k} \frac{1}{\lambda_{\mu}^{k}} u_{\alpha^{\prime} \mu}^{k}
$$

Let us also use new variables $x_{k}^{\alpha}=-\sum_{\eta} u_{\alpha \eta}^{k} w_{\eta}^{k}$ (so $z_{\alpha}=y_{k}^{\alpha}+x_{k}^{\alpha}$ ). As a result, formula (19) for $\left\langle D_{k}^{\alpha}\right\rangle$ can be written in the final form

$$
\left\langle D_{k}^{\alpha}\right\rangle=\overline{b_{k}^{\alpha}\left(y_{k}^{x}, y_{k}^{y}\right)}
$$

where the line over an expression means its averaging over the Gaussian fluctuations

$$
\overline{F_{k}}=\frac{1}{4 \pi \sqrt{\mathcal{D}_{k}}} \int_{-\infty}^{+\infty} \exp \left(-\frac{1}{4} \sum_{\alpha \alpha^{\prime}} A_{k k}^{\alpha \alpha^{\prime}} x_{k}^{\alpha} x_{k}^{\alpha^{\prime}}\right) F_{k}\left(y_{k}^{x}+x_{k}^{x}, y_{k}^{y}+x_{k}^{y}\right) \mathrm{d} x_{k}^{x} \mathrm{~d} x_{k}^{y} .
$$

Now we consider the contribution of two-tailed parts. In general, a two-tailed part contains, as the intermediate structure element, the full semi-invariant pair correlation function [10]:

$$
\Upsilon=\{3
$$


or

$$
\tilde{I}_{k k}^{\alpha_{1} \alpha_{2}}(\mathbf{q})=\beta^{2} \sum_{k^{\prime} \gamma_{1} \gamma_{2}} \psi_{k k^{\prime}}^{\alpha_{1} \gamma_{1}}(\mathbf{q}) M_{k^{\prime} k^{\prime}}^{\gamma_{1} \gamma_{2}} \psi_{k^{\prime} k}^{\gamma_{2} \alpha_{2}}(\mathbf{q}),
$$

where $\psi_{k k^{\prime}}^{\alpha \alpha^{\prime}}(\mathbf{q})$ are Fourier transforms of the interaction constants and $M_{k k}^{\gamma_{1} \gamma_{2}}$ are the pair correlators constructed on the $D_{k}^{\alpha}$ operators

$$
M_{k k}^{\gamma_{1} \gamma_{2}}=\left\langle D_{k}^{\gamma_{1}} D_{k}^{\gamma_{2}}\right\rangle^{\mathrm{c}}
$$

In the Onyszkiewicz approximation the correlator $M_{k k}^{\alpha_{1} \alpha_{2}}$ is given by the series, which is analogous to expression (9). The semi-invariants calculated in the MFA (corresponding to the simple ovals) are renormalized in a similar way by the fluctuation contributions of two-tailed parts [8]:

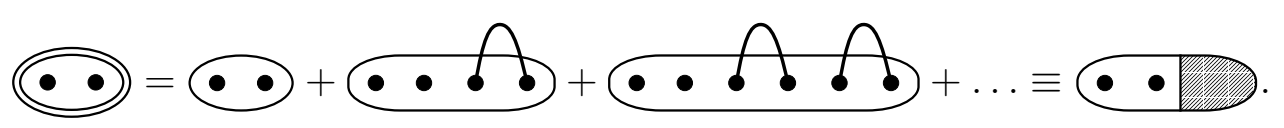

A respective analytic expression, which can be obtained by the procedure similar to the described above, is as follows:

$$
M_{k k}^{\alpha_{1} \alpha_{2}}=\overline{b_{k \alpha_{1} \alpha_{2}}^{[1]}\left(y_{k}^{x}, y_{k}^{y}\right)}
$$

where

$$
b_{k \alpha_{1} \alpha_{2}}^{[1]}\left(y_{k}^{x}, y_{k}^{y}\right)=\frac{\partial^{2} L_{k}}{\partial\left(\beta y_{k}^{\alpha_{1}}\right) \partial\left(\beta y_{k}^{\alpha_{2}}\right)} .
$$

Relations (22), (25) and (28) form a set of integral equations determining both equilibrium value of average dipole moments $\left\langle D_{k}^{\alpha}\right\rangle$ and the variables $\lambda_{\mu}^{k}$ proportional to the root-mean-square (r.m.s.) deviations of the respective Gaussian fluctuations.

\section{Free energy in Gaussian approximation}

The set of the self-consistency equations for the average values (and their r.m.s. deviations in the case of the Gaussian approximation) is a necessary tool for the study of thermodynamics of the model. The separation of solutions of the set of equations (22), (25) and (28), which correspond to the thermodynamically stable equilibrium state of the system, can be performed using the usual criterion of the minimum value of the free energy (calculated in the same approximation).

In our case we need to generalize the procedure of constructing the appropriate expression for this function given in [8]. The required formula for the free energy $F$ must lead to equations (22), (25) and (28) by satisfying the stationary conditions

$$
\begin{aligned}
& \partial(F / N) / \partial\left\langle D_{k}^{\alpha}\right\rangle=0, \\
& \partial(F / N) / \partial j_{k k}^{\alpha_{1} \alpha_{2}}=0,
\end{aligned}
$$

where the $\left\langle D_{k}^{\alpha}\right\rangle$ averages and $j_{k k}^{\alpha_{1} \alpha_{2}}$ r.m.s. deviations are considered to be independent variables; it should also go over into the MFA expression when the contributions 
connected with the two-tailed parts are neglected. Such a form in the Onyszkiewicz approach for the model under investigation is given by

$$
\begin{aligned}
F / N= & \frac{1}{2} \sum_{k k^{\prime}} \sum_{\alpha \alpha^{\prime}}\left\langle D_{k}^{\alpha}\right\rangle \psi_{k k^{\prime}}^{\alpha \alpha^{\prime}}\left\langle D_{k^{\prime}}^{\alpha^{\prime}}\right\rangle-\Theta \sum_{k} \overline{L_{k}\left(y_{k}^{x}, y_{k}^{y}\right)} \\
& +\frac{1}{2 \beta} \sum_{k k^{\prime}} \sum_{\alpha_{1} \alpha_{2}} \sum_{\gamma_{1} \gamma_{2}} j_{k k}^{\alpha_{1} \alpha_{2}}\left(\hat{S}^{-1}\right)_{k, k^{\prime}}^{\alpha_{1} \alpha_{2}, \gamma_{1} \gamma_{2}} j_{k^{\prime} k^{\prime}}^{\gamma_{1} \gamma_{2}} .
\end{aligned}
$$

Here the matrix

$$
S_{k, k^{\prime}}^{\alpha_{1} \alpha_{2}, \gamma_{1} \gamma_{2}}=\frac{1}{N} \sum_{\mathbf{q}} \psi_{k k^{\prime}}^{\alpha_{1} \gamma_{1}}(\mathbf{q}) \psi_{k^{\prime} k}^{\gamma_{2} \alpha_{2}}(\mathbf{q}) \quad\left(S_{k^{\prime}, k}^{\gamma_{1} \gamma_{2}, \alpha_{1} \alpha_{2}}=S_{k, k^{\prime}}^{\alpha_{1} \alpha_{2}, \gamma_{1} \gamma_{2}}\right)
$$

is introduced (its structure is considered in appendix A).

The bar over the expression in the second term of (32) denotes its average with the Gaussian distribution. In fact, the MFA form [5] could be obtained neglecting both the last term and the averaging over fluctuation in the second one.

It is easy to see that the expression (32) satisfies the condition (30)

$$
\begin{aligned}
\partial(F / N) / \partial\left\langle D_{k}^{\alpha}\right\rangle & =\sum_{k^{\prime} \alpha^{\prime}} \psi_{k k^{\prime}}^{\alpha \alpha^{\prime}}\left\langle D_{k^{\prime}}^{\alpha^{\prime}}\right\rangle-\sum_{k^{\prime} \alpha^{\prime}} \psi_{k k^{\prime}}^{\alpha \alpha^{\prime}} \frac{\partial \overline{L_{k^{\prime}}}}{\partial\left(\beta y_{k^{\prime}}^{\alpha^{\prime}}\right)} \\
& =\sum_{k^{\prime} \alpha^{\prime}} \psi_{k k^{\prime}}^{\alpha \alpha^{\prime}}\left(\left\langle D_{k^{\prime}}^{\alpha^{\prime}}\right\rangle-\overline{b_{k^{\prime} \alpha^{\prime}}}\right)=0
\end{aligned}
$$

when relation (22) is fulfilled.

The proof of the fulfillment of condition (31) is more complicated. This condition can be written as

$$
\frac{\partial(F / N)}{\partial j_{k k}^{\alpha_{1} \alpha_{2}}}=\frac{1}{\beta} \sum_{k^{\prime} \gamma_{1} \gamma_{2}}\left(\hat{S}^{-1}\right)_{k, k^{\prime}}^{\alpha_{1} \alpha_{2}, \gamma_{1} \gamma_{2}} j_{k^{\prime} k^{\prime}}^{\gamma_{1} \gamma_{2}}-\Theta \frac{\partial}{\partial j_{k k}^{\alpha_{1} \alpha_{2}}} \overline{L_{k}\left(y_{k}^{x}, y_{k}^{y}\right)}=0
$$

Making use of relation

$$
j_{k k}^{\alpha_{1} \alpha_{2}}=\beta^{2} \sum_{k^{\prime} \gamma_{1} \gamma_{2}} S_{k, k^{\prime}}^{\alpha_{1} \alpha_{2}, \gamma_{1} \gamma_{2}} M_{k^{\prime} k^{\prime}}^{\gamma_{1} \gamma_{2}}
$$

one can simplify the first term in (35) and the condition becomes much more compact

$$
M_{k k}^{\alpha_{1} \alpha_{2}}=\frac{1}{\beta^{2}} \frac{\partial}{\partial j_{k k}^{\alpha_{1} \alpha_{2}}} \overline{L_{k}\left(y_{k}^{x}, y_{k}^{y}\right)}
$$

Applying the following identities

$$
\frac{\partial}{\partial j_{k k}^{\alpha_{1} \alpha_{2}}} \frac{1}{\sqrt{\mathcal{D}_{k}}}=-\frac{1}{2 \sqrt{\mathcal{D}_{k}}} A_{k k}^{\alpha_{2} \alpha_{1}}
$$

and

$$
\partial A_{k k}^{\gamma_{1} \gamma_{2}} / \partial j_{k k}^{\alpha_{1} \alpha_{2}}=-A_{k k}^{\gamma_{1} \alpha_{1}} A_{k k}^{\alpha_{2} \gamma_{2}}
$$


(see the proof in appendix B) the derivative with respect to $j_{k k}^{\alpha_{1} \alpha_{2}}$ could be taken as

$$
\begin{aligned}
\frac{\partial \overline{L_{k}}}{\partial j_{k k}^{\alpha \alpha^{\prime}}}= & \frac{1}{4 \pi \sqrt{\mathcal{D}_{k}}} \int_{-\infty}^{+\infty} \int_{-\infty}\left(-\frac{1}{2} A_{k k}^{\alpha_{2} \alpha_{1}}+\frac{1}{4} \sum_{\gamma_{1} \gamma_{2}} A_{k k}^{\gamma_{1} \alpha_{1}} A_{k k}^{\alpha_{2} \gamma_{2}} x_{k}^{\gamma_{1}} x_{k}^{\gamma_{2}}\right) \\
& \times \exp \left(-\frac{1}{4} \sum_{\alpha \alpha^{\prime}} A_{k k}^{\alpha \alpha^{\prime}} x_{k}^{\alpha} x_{k}^{\alpha^{\prime}}\right) L_{k}\left(y_{k}^{x}+x_{k}^{x}, y_{k}^{y}+x_{k}^{y}\right) \mathrm{d} x_{k}^{x} \mathrm{~d} x_{k}^{y}
\end{aligned}
$$

On the other hand, following definition (28) one can write down

$$
M_{k k}^{\alpha_{1} \alpha_{2}}=\frac{1}{4 \pi \sqrt{\mathcal{D}_{k}}} \int_{-\infty}^{+\infty} \exp \left(-\frac{1}{4} \sum_{\alpha \alpha^{\prime}} A_{k k}^{\alpha \alpha^{\prime}} x_{k}^{\alpha} x_{k}^{\alpha^{\prime}}\right) \frac{1}{\beta^{2}} \frac{\partial^{2} L_{k}}{\partial x_{k}^{\alpha_{1}} \partial x_{k}^{\alpha_{2}}} \mathrm{~d} x_{k}^{x} \mathrm{~d} x_{k}^{y} .
$$

After that, integrating twice by parts in (41), we obtain

$$
\begin{aligned}
M_{k k}^{\alpha_{1} \alpha_{2}}= & \frac{1}{\beta^{2}} \frac{1}{4 \pi \sqrt{\mathcal{D}_{k}}} \int_{-\infty}^{+\infty}\left[\frac{1}{2} \exp \left(-\frac{1}{4} \sum_{\alpha \alpha^{\prime}} A_{k k}^{\alpha \alpha^{\prime}} x_{k}^{\alpha} x_{k}^{\alpha^{\prime}}\right) \sum_{\gamma_{2}} A_{k k}^{\alpha_{2} \gamma_{2}} x_{k}^{\gamma_{2}}\right] \frac{\partial L_{k}}{\partial x_{k}^{\alpha_{1}}} \mathrm{~d} x_{k}^{x} \mathrm{~d} x_{k}^{y} \\
= & \frac{1}{\beta^{2}} \frac{1}{4 \pi \sqrt{\mathcal{D}_{k}}} \int_{-\infty}^{+\infty}\left(-\frac{1}{2} A_{k k}^{\alpha_{2} \alpha_{1}}+\frac{1}{4} \sum_{\gamma_{1} \gamma_{2}} A_{k k}^{\gamma_{1} \alpha_{1}} A_{k k}^{\alpha_{2} \gamma_{2}} x_{k}^{\gamma_{1}} x_{k}^{\gamma_{2}}\right) \\
& \times \exp \left(-\frac{1}{4} \sum_{\alpha \alpha^{\prime}} A_{k k}^{\alpha \alpha^{\prime}} x_{k}^{\alpha} x_{k}^{\alpha^{\prime}}\right) L_{k}\left(y_{k}^{x}+x_{k}^{x}, y_{k}^{y}+x_{k}^{y}\right) \mathrm{d} x_{k}^{x} \mathrm{~d} x_{k}^{y} .
\end{aligned}
$$

The last expression coincide with the derivative $\partial \overline{L_{k}} / \partial j_{k k}^{\alpha \alpha^{\prime}}$ (formula (40)). It is the evidence of the fulfillment of the condition (37) and thereby of satisfying the equation (31).

Hence, expression (32) for the free energy, which satisfies the necessary conditions, can be used for the analysis of equilibrium states of the four-state model. It should be mentioned that in the diagrammatic representation the fluctuation part of this expression corresponds to the sum of the ring diagrams created by two-tailed parts (this question is analyzed more in details in [11] on the example of the pseudospin system with the indirect interaction).

\section{Thermodynamics of the phase transitions in DMAGaS- DMAAIS ferroelectrics (numerical analysis)}

The results obtained in the previous section can be illustrated on the example of DMAGaS-DMAAlS ferroelectrics. Unfortunately, the set of equations (22), (25) and (28) is itself a sophisticated challenge for numerical methods and in the most interesting region in the vicinity of triple and tricritical points a problem is further complicated due to the interplay between different orderings as it will be shown below. So our study is limited only to the case of the sufficiently long-ranged interaction 


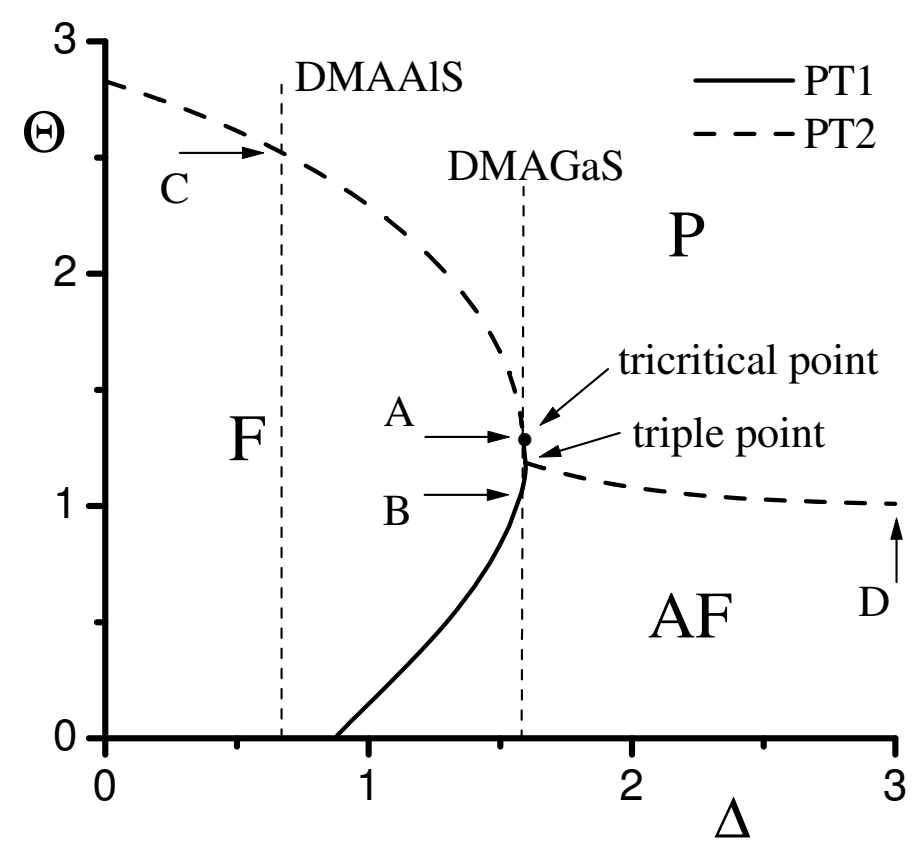

Figure 1. Phase diagram $\Theta-\Delta$; solid and dashed lines indicate the first order and the second order phase transitions, respectively.

between DMA groups. Nevertheless, the obtained results allow one to estimate the relative magnitude of the Gaussian fluctuations. At small values of structure factors $\left(\gamma_{1}=\gamma_{2}=1 \times 10^{-5}\right)$ the phase diagram obtained in MFA [5] remains practically unchanged, so it is used as a starting point for further study (figure 1). In all figures parameters of the dipole-dipole interaction are made dimensionless by normalization on $(a-d)$ with the following values: $a=0.7, b=2.075, d=-0.3, e=-0.525$, $g=1.05, k=-0.55, d_{y} / d_{x}=1.4$. Such a set of numerical values of interaction
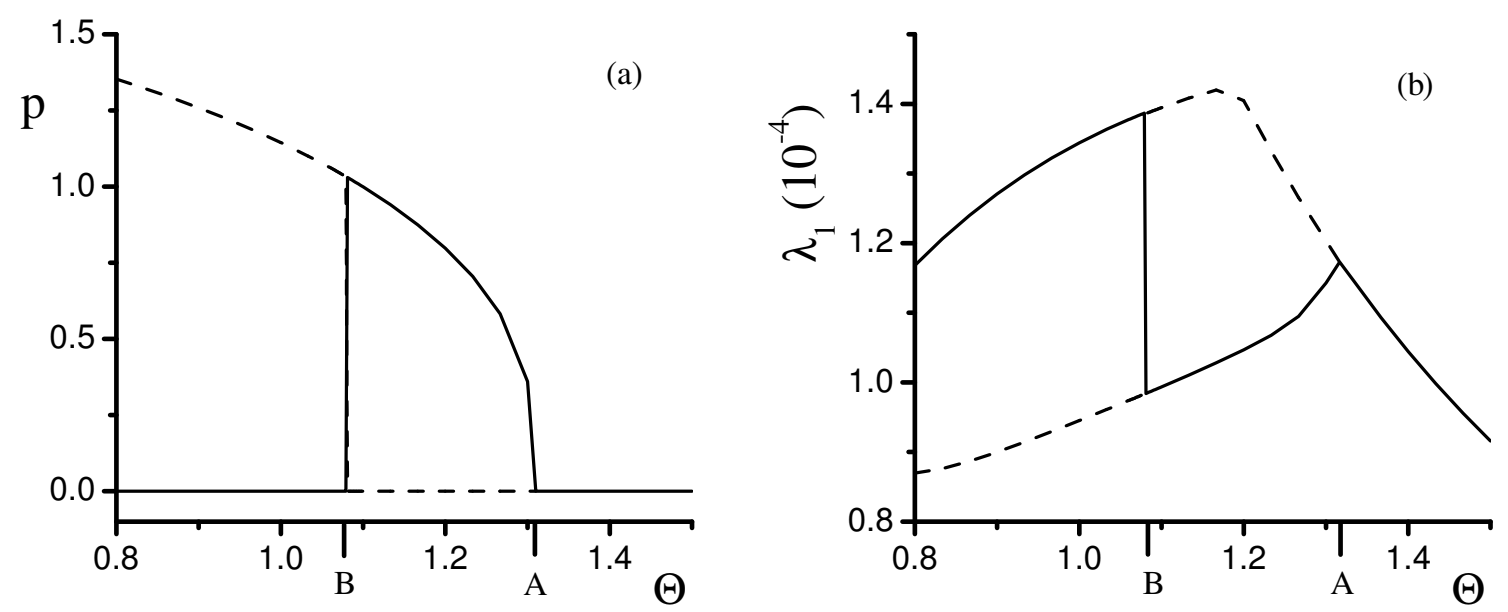

Figure 2. Temperature dependences of polarization (a) and the r.m.s. variation of fluctuations (b) for the DMAGaS-like case $(\Delta=1.5855)$; solid and dashed lines indicate thermodynamically stable and unstable solutions, respectively. 

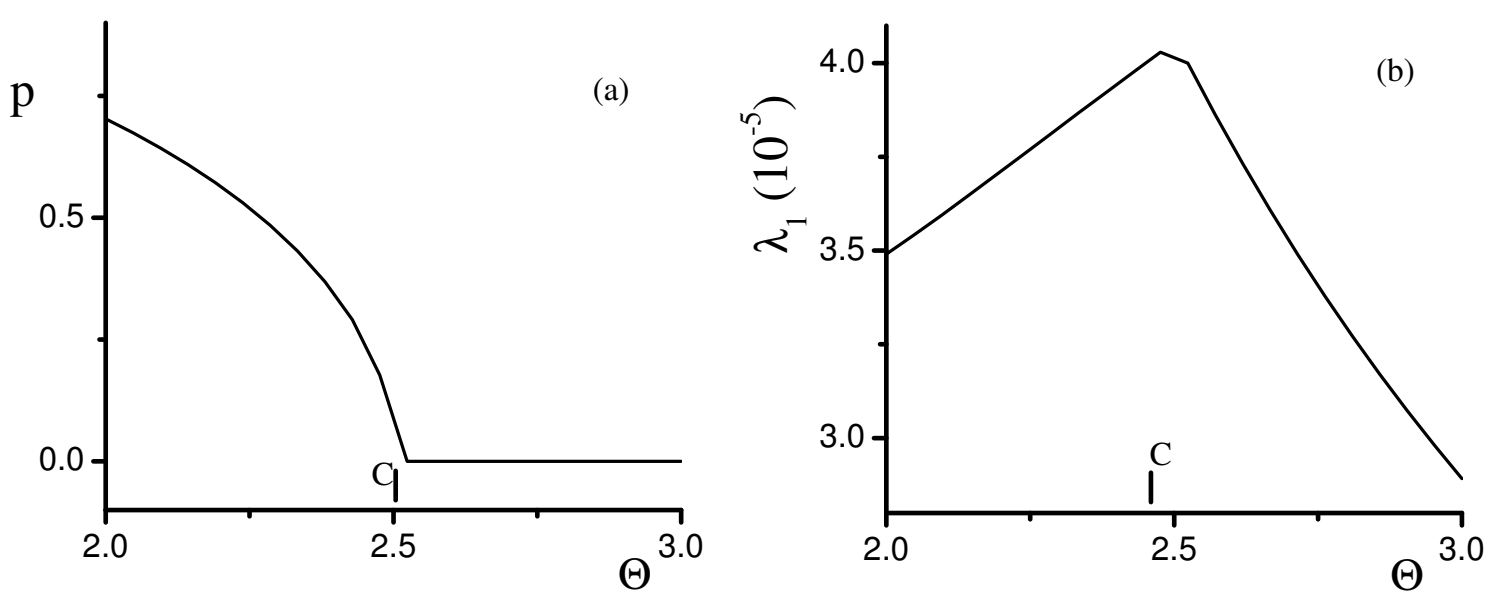

Figure 3. Temperature dependences of polarization (a) and the r.m.s. variation of fluctuations (b) for the DMAAlS-like case $(\Delta=0.7)$.

constants was used in paper [1] in the description of thermodynamics of the considered family of crystals in the MFA and was obtained by fitting the results of the theoretical calculations to the experimental data. The difference $\Delta$ between the site energies as well as temperature $\Theta$ are given in $(a-d) d_{x}^{2}$ units while the variable $\lambda_{1}$ characterizing the r.m.s. deviation of fluctuations is counted in $(a-d) d_{x}$ units.

In the case typical of the DMAGaS crystal both the phase transitions (the points $\mathrm{A}$ and $\mathrm{B}$ in figure 1) in the sequence of para-ferro-antiferroelectric phases take place in a close proximity to the triple and tricritical points. Figure 2a illustrates temperature dependence of the order parameter $p=\left\langle D_{1}^{x}\right\rangle+\left\langle D_{2}^{x}\right\rangle$ which is proportional to the $x$-component of the polarization of the system $P_{x}=p / v_{\mathrm{c}}$, where $v_{\mathrm{c}}$ is the volume of a unit cell. Usually the Gaussian fluctuations do not play an important role for the first order transitions (except those close to the second order transitions). But in our case the fluctuations are of the same order in both points of
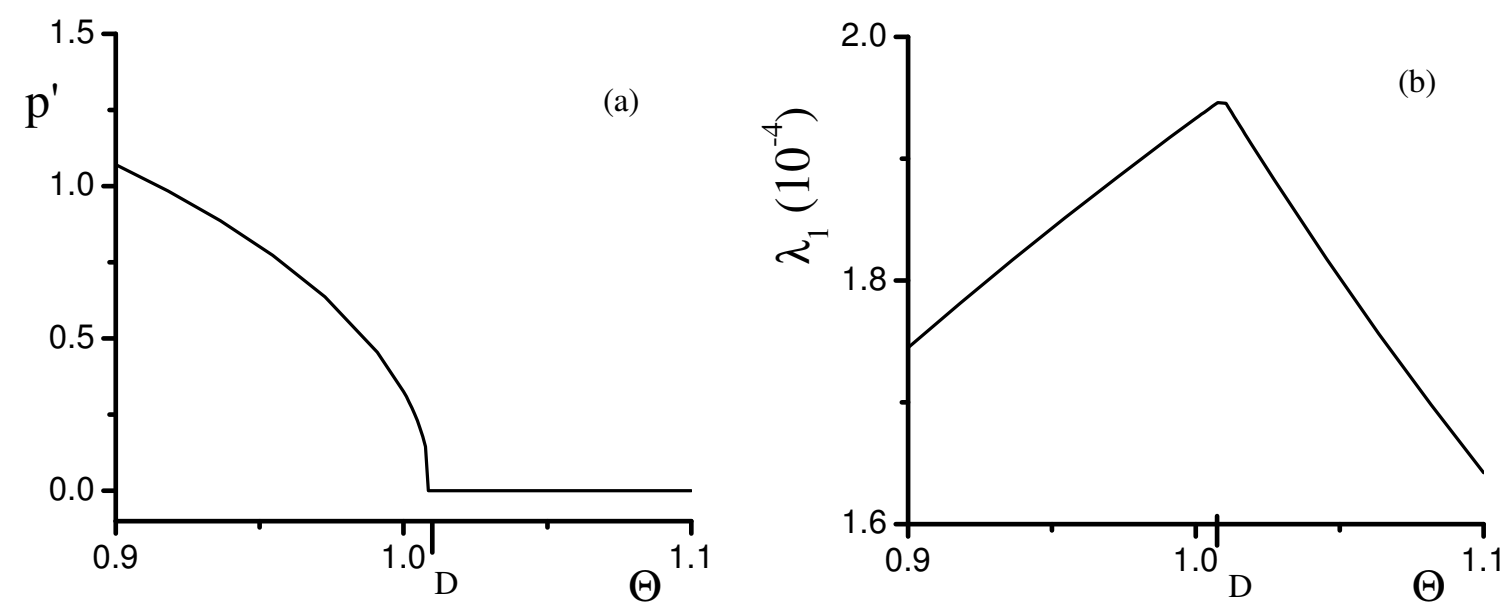

Figure 4. Temperature dependences of antipolarization (a) and the r.m.s. variation of fluctuations (b) for the Ising-like case $(\Delta=3)$. 
phase transitions (figure $2 \mathrm{~b}$ ). The corresponding $\lambda_{1}$ variable (which is the maximum eigenvalue of the matrix $\hat{J}_{k}$ ) describes fluctuations of both ferroelectric and antiferroelectric order parameters with their nearly equal contributions. Such a behaviour has a simple explanation: the true first order transition in the point $\mathrm{B}$ is very close to the point of instability of paraelectric phase with respect to the appearance of antiferroelectric state. The corresponding transformation is not of the second order but the magnitude of fluctuations significantly increases in the transition point. In other words, near the triple point the Gaussian fluctuations play an important role even at the first order phase transitions. Such a mutual influence of different orderings greatly complicates the numerical analysis.

It is interesting to compare the behaviour of fluctuations far from the triple point for other different cases: the DMAAlS-like one (the point $\mathrm{C}$ in figure 1), where all four orientational states have nonzero occupation, and the Ising-like case (the point D ibidem), where only one pair of states is occupied. As one can see in figures 3 and 4 , the behaviour of the order parameters $\left(p\right.$ and $p^{\prime}=\left\langle D_{1}^{x}\right\rangle-\left\langle D_{2}^{x}\right\rangle$ which has the meaning of antipolarization) and the Gaussian fluctuation parameters are very similar in both cases and rather usual for the second order phase transitions (see, for example, [10]).

\section{Conclusions}

The present paper extends the field of application of the Onyszkiewicz method of describing of the Gaussian fluctuations of polarization from the Ising-type (two-level) models to the multi-level models with pair-wise interaction of particles. We develop a thermodynamically consistent procedure to determine the order parameters (the components of the dipole moments) and the r.m.s. variations of fluctuations as functions of temperature. Ferroelectric crystals of the DMAGaS-DMAAlS family, where the adequate description of the sequence of phase transitions between para-, ferro- and antiferroelectric phases was obtained by means of the four-site model (such a model considers possible orientational states of the DMA groups), serve as an example of practical utilization of our scheme.

The phase diagram for the considered system is obtained in the MFA corrected by the allowance for fluctuations. At the sufficiently long-ranged interaction between particles, it successfully reproduces the experimentally observed sequences of phase transitions both for DMAGaS and DMAAlS at different values of parameters. The r.m.s. variations of the Gaussian fluctuations of the order parameters have a temperature behaviour typical of the second order phase transitions in the Ising model [8] (the maximum is reached in the phase transition point) far from the triple and tricritical points. In the vicinity of the triple point, fluctuations grow up for both ferroelectric and antiferroelectric states, so the r.m.s. variations are of the same order for all types of phase transitions. However, in the case of the first order transition they demonstrate a jump-like behaviour in the phase transition point while for the second order transitions a peak-like dependence takes place.

At higher values of $\gamma_{1}$ and $\gamma_{2}$ parameters (less long-ranged interaction) one should 
expect more pronounced changes of the phase diagram $(T, \Delta)$ (lowering of temperatures of phase transitions, especially for the second order ones) and the respective increase of the r.m.s. variation $\lambda_{1}$. This case needs a more detailed study but the conclusion about a near equivalent role of fluctuations for all the transitions placed near the triple point of the mentioned diagram seems to be still valid.

\section{Acknowledgements}

This work was partially supported by the Fundamental Researches Fund of the Ministry of Ukraine of Science and Education (Project No. 02.07/00310).

\section{A. Matrix of effective interactions}

The Fourier transform of the matrix of dipole-dipole interactions can be simplified in the spirit of the MFA

$$
\begin{aligned}
\psi_{k k^{\prime}}^{\alpha \alpha^{\prime}}(\mathbf{q}) & =\sum_{n-n^{\prime}} \Psi_{k k^{\prime}}^{\alpha \alpha^{\prime}}\left(n n^{\prime}\right) \mathrm{e}^{\mathrm{i} \mathbf{q}\left(\mathbf{R}_{n k}-\mathbf{R}_{n^{\prime} k^{\prime}}\right)} \\
& =\psi_{k k^{\prime}}^{\alpha \alpha^{\prime}} \frac{1}{N} \sum_{n-n^{\prime}} \mathrm{e}^{\mathrm{i} \mathbf{q}\left(\mathbf{R}_{n k}-\mathbf{R}_{n^{\prime} k^{\prime}}\right)}
\end{aligned}
$$

The following notation is assigned to the momentum dependent factor in the expression above

$$
\gamma_{k k^{\prime}}(\mathbf{q})=\frac{1}{N} \sum_{n-n^{\prime}} \mathrm{e}^{\mathrm{i} \mathbf{q}\left(\mathbf{R}_{n k}-\mathbf{R}_{n^{\prime} k^{\prime}}\right)}, \quad \gamma_{k k^{\prime}}(-\mathbf{q})=\gamma_{k^{\prime} k}(\mathbf{q})
$$

There are only two various combinations of such factors in the matrix $\left[S_{k, k^{\prime}}^{\alpha_{1} \alpha_{2}, \gamma_{1} \gamma_{2}}\right]$

$$
\gamma_{1}=\frac{1}{N} \sum_{\mathbf{q}} \gamma_{11}^{2}(\mathbf{q}), \quad \gamma_{2}=\frac{1}{N} \sum_{\mathbf{q}} \gamma_{12}(\mathbf{q}) \gamma_{21}(\mathbf{q})
$$

Using the definition

$$
S_{k, k^{\prime}}^{\alpha_{1} \alpha_{2}, \gamma_{1} \gamma_{2}}=\frac{1}{N} \sum_{\mathbf{q}} \psi_{k k^{\prime}}^{\alpha_{1} \gamma_{1}}(\mathbf{q}) \psi_{k^{\prime} k}^{\gamma_{2} \alpha_{2}}(\mathbf{q})
$$

one can write down the explicit form of the matrix

$$
\hat{S}=\left(\begin{array}{rrrrrrrr}
a^{2} \gamma_{1} & a b \gamma_{1} & a b \gamma_{1} & b^{2} \gamma_{1} & d^{2} \gamma_{2} & d e \gamma_{2} & d e \gamma_{2} & e^{2} \gamma_{2} \\
a b \gamma_{1} & a g \gamma_{1} & b^{2} \gamma_{1} & b g \gamma_{1} & -d e \gamma_{2} & d k \gamma_{2} & -e^{2} \gamma_{2} & e k \gamma_{2} \\
a b \gamma_{1} & b^{2} \gamma_{1} & a g \gamma_{1} & b g \gamma_{1} & -d e \gamma_{2} & -e^{2} \gamma_{2} & d k \gamma_{2} & e k \gamma_{2} \\
b^{2} \gamma_{1} & b g \gamma_{1} & b g \gamma_{1} & g^{2} \gamma_{1} & e^{2} \gamma_{2} & -e k \gamma_{2} & -e k \gamma_{2} & k^{2} \gamma_{2} \\
d^{2} \gamma_{2} & -d e \gamma_{2} & -d e \gamma_{2} & e^{2} \gamma_{2} & a^{2} \gamma_{1} & -a b \gamma_{1} & -a b \gamma_{1} & b^{2} \gamma_{1} \\
d e \gamma_{2} & d k \gamma_{2} & -e^{2} \gamma_{2} & -e k \gamma_{2} & -a b \gamma_{1} & a g \gamma_{1} & b^{2} \gamma_{1} & -b g \gamma_{1} \\
d e \gamma_{2} & -e^{2} \gamma_{2} & d k \gamma_{2} & -e k \gamma_{2} & -a b \gamma_{1} & b^{2} \gamma_{1} & a g \gamma_{1} & -b g \gamma_{1} \\
e^{2} \gamma_{2} & e k \gamma_{2} & e k \gamma_{2} & k^{2} \gamma_{2} & b^{2} \gamma_{1} & -b g \gamma_{1} & -b g \gamma_{1} & g^{2} \gamma_{1}
\end{array}\right) .
$$




\section{B. Some relations for inverse matrices}

Let $\hat{A}$ be a nonsingular matrix. Then

$$
A_{i k}=(-1)^{i+k} D_{i k}=\partial \mathcal{D} / \partial a_{i k}
$$

where $A_{i k}$ and $D_{i k}$ are the algebraic complement and the minor of the element $a_{i k}$, respectively, $\mathcal{D}$ is the determinant of the matrix $\hat{A}$. Let $\hat{A}^{-1}$ be the matrix inverse to the $\hat{A}$

$$
\hat{A}^{-1} \hat{A}=\hat{A} \hat{A}^{-1}=\hat{I}
$$

where $\hat{I}$ is a unit matrix;

$$
\left(\hat{A}^{-1}\right)_{i k}=A_{k i} / \mathcal{D}
$$

and

$$
\left(\hat{A}^{-1} \hat{A}\right)_{k l}=\frac{1}{\mathcal{D}} \sum_{i} A_{i k} a_{i l}=\delta_{k l} .
$$

Let us take the partial derivative of the above expression with respect to an element of the matrix $\hat{A}$

$$
\frac{\partial}{\partial a_{m n}} \sum_{i}\left(\hat{A}^{-1}\right)_{k i} a_{i l}=\sum_{i}\left[\frac{\partial}{\partial a_{m n}}\left(\hat{A}^{-1}\right)_{k i}\right] a_{i l}+\sum_{i}\left(\hat{A}^{-1}\right)_{k i} \frac{\partial a_{i l}}{\partial a_{m n}} .
$$

After the multiplication of both sides by $\left(\hat{A}^{-1}\right)_{l i}$ and the subsequent summation over $j$ one can obtain such a relation

$$
\sum_{i l}\left[\frac{\partial}{\partial a_{m n}}\left(\hat{A}^{-1}\right)_{k i}\right] a_{i l}\left(\hat{A}^{-1}\right)_{l j}+\sum_{i l}\left(\hat{A}^{-1}\right)_{k i} \delta_{i m} \delta_{l n}\left(\hat{A}^{-1}\right)_{l j}=0
$$

resulting in the final formula

$$
\frac{\partial}{\partial a_{m n}}\left(\hat{A}^{-1}\right)_{k j}=-\left(\hat{A}^{-1}\right)_{k m}\left(\hat{A}^{-1}\right)_{n j}
$$

\section{References}

1. Stasyuk I.V., Velychko O.V. // Journ. Phys. Studies, 2000, vol. 4, p. 92-99.

2. Pietraszko A., Łukaszewicz K, Kirpicznikowa L.F. // Polish J. Chem., 1993, vol. 67, p. $1877-1884$.

3. Pietraszko A., Łukaszewicz K. // Polish J. Chem., 1994, vol. 68, p. 1239-1243.

4. Pietraszko A., Łukaszewicz K., Kirpicznikowa L.F. // Polish J. Chem., 1995, vol. 69, p. $922-930$.

5. Stasyuk I.V., Velychko O.V. // Phase transitions, 2001, vol. 73, p. 483-501.

6. Kapustianik V., Sveleba S., Stasyuk I., Velychko O., Czapla Z., Tchukvinskyi R. // Phys. status solidi (b), 2001, vol. 228, p. 785-798.

7. Yasuda N., Kaneda A., Czapla Z. // Ferroelectrics, 1999, vol. 223, p. 71.

8. Onyszkiewicz Z., Wierzbicki A. // Physica B, 1988, vol. 151, p. 462-474. 
9. Stasyuk I.V., Slobodyan P.M. // Theor. Math. Phys., 1974, vol. 19, No. 3, p. 423-428 (in Russian).

10. Izyumov Yu.A., Kassan-Ogly F.A., Skryabin Yu.N. Field Methods in the Theory of Ferromagnetism. Moscow, Nauka, 1974 (in Russian).

11. Stasyuk I.V., Tabunshchyk K.V. // Condens. Matter Phys., 2001, vol. 4, No. 1(25), p. 109-118.

\title{
Гаусові флуктуації поляризації в області фазових переходів у сегнетоелектриках DMAGaS-DMAAIS у рамках чотиристанової моделі
}

\author{
І.В.Стасюк, О.В.Величко \\ Інститут фізики конденсованих систем НАН України, \\ 79011 Львів, вул. Свєнціцького, 1 \\ Отримано 12 листопада 2003 р.
}

Представлено вдосконалений опис термодинаміки сегнетоелектриків сімейства DMAGaS-DMAAIS, що враховує гаусові флуктуації поляризації. Їх роль зростає в околі фазових переходів другого (або першого, близького до другого) роду, що мають місце в згаданих кристалах. Теорія, побудована шляхом узагальнення наближення Онишкевича для випадку чотиристанової моделі, дає прості за формою і чіткі за фізичним змістом вирази для компонент поляризації, ї флуктуацій та вільної енергії. Отримані числовим способом результати показують, що у досліджуваній системі гаусові флуктуації, приводячи при достатньо далекосяжній взаємодії до незначного пониження температури фазових переходів, в околі потрійної точки $€$ однакового порядку величини при переходах як першого, так і другого роду.

Ключові слова: сегнетоелектрики, DMAGaS, DMAAIS, мікроскопічна модель, гаусові флуктуації

PACS: 77.84.-s, 64.60.Cn 
